\title{
Therapeutic Consideration of Periappendiceal Abscess: an Evaluation of Non-surgical Treatment Followed by Minimally Invasive Interval Appendectomy
}

\author{
Yeong-Soo Jo, M.D., Song-Soo Yang, M.D., Yeong-Chul Im, M.D., Dong-Jin Park, M.D., Gyu-Yeol Kim, M.D. \\ Department of Surgery, University of Ulsan College of Medicine and Ulsan University Hospital, Ulsan, Korea
}

Purpose: Periappendiceal abscess (PAA) is a severe complicated appendicitis with high morbidity. Non-surgical treatment followed by interval appendectomy (IA) is associated with lower complication rate compared with emergency surgery (ES) and minimally invasive surgery (MIS) can be done more often. The purpose of this study is to assess the incidence and factors predictive of complications of surgery for PAA and to evaluate the clinical effectiveness of IA as a treatment policy to increase MIS.

Methods: Retrospectively, we reviewed 171 patients undergoing surgery for PAA between 2011 and 2016 at Ulsan University Hospital. The incidence and influence of different factors were assessed by univariate and multivariate analyses.

Results: In 171 patients, 28 (16.4\%) developed postoperative complications, which included; wound complications (7.6\%), intra-abdominal abscess (4.1\%) and ileus (2.9\%). In both analyses, only ES was independently associated with postoperative complications; (Relative risk, 15.0; 95\% Confidence interval, 2.4 92.5). Comparing the IA and ES groups revealed that operative time, complication rate, laparoscopic approach, postoperative hospitalization, postoperative antibiotic use and bowel resection rate were significantly different. The postoperative complication rate of patients with PAA in ES group was $28.7 \%$, which was statistically higher than that of IA group (3.6\%). Especially, MIS rate was more than 9 -times greater in the IA group (98.8\% vs. $10.3 \%, p<0.001)$. Although the IA group required additional hospitalization, there was no statistical difference between the two groups in total length of hospital stay.

Conclusion: Morbidity was high for patients who had emergency surgery for PAA. ES was the only factor associated with postoperative morbidity. IA can reduce the postoperative complication rate and allowed MIS to be used more often as a useful treatment policy for PAA.

Keywords: Periappendiceal abscess, Non-surgical treatment, Minimal invasive surgery, Interval appendectomy

This is an Open Access article distributed under the terms of the Creative Commons Attribution Non-Commercial License (http:// creativecommons.org/licenses/by-nc/4.0/) which permits unrestricted non-commercial use, distribution, and reproduction in any medium, provided the original work is properly cited.
Received July 27, 2017

Revised 1st September 14, 2017 2nd October 8, 2017

Accepted October 10, 2017

Corresponding author

Song-Soo Yang

Department of Surgery, Ulsan

University Hospital, 290-3 Jeonhadong, Dong-gu, Ulsan 44033, Korea Tel: $+82-52-250-7722$

Fax: +82-52-250-7350

E-mail: pau1913@hanmail.net

\section{INTRODUCTION}

Acute appendicitis should be considered first for adult or pediatric patients who present with acute abdominal symp- toms. Most will need surgery. A meta-analysis of 61 observational studies in which periappendiceal abscess (PAA) or phlegmon was present in $3.8 \%$ of patients with appendicitis found that emergency surgery (ES) as associated with mor- 
bidity in $35.6 \%$ compared with that in $13.5 \%$ with nonsurgical treatment, which has considerable influence on treatment policy and cost remarkably. ${ }^{1}$

Conservative management using antibiotics alone and image-guided percutaneous drainage for patients with PAA has achieved high success rates of $76 \%$ to $97 \%$, with a low incidence of complications. ${ }^{2-8}$

After achieving complete resolution of the inflammatory mass with non-surgical treatment, interval appendectomy (IA) is a less hazardous and less challenging operation, compared with ES. However, IA requires extra hospitalization for surgery and can take a long time from the time of illness to the end of treatment. ${ }^{9,10}$

In this study, we tried to assess the incidence and the type of postoperative complications in patients who underwent surgery for PAA, and evaluated the risk factors of postoperative morbidity. In addition, we explored the clinical usefulness of non-surgical treatment followed by IA to increase the rate of minimally invasive surgery (MIS).

\section{MATERIALS AND METHODS}

A total of 2,392 patients underwent a surgery for appendicitis at OOO Hospital between January 2010 and May 2016. Of these 2,392 patients, we identified 180 consecutive patients who underwent surgery for established acute appendicitis accompanied by PAA and retrospectively reviewed our prospectively collected database of patients with PAA. We excluded patients diagnosed as malignancy (1), who were pregnant (1), or received other combined operations (3) and already had Crohn's disease (4). Finally, total of 171 patients met the study criteria (Fig. 1). The $O 00$ Hospital Institutional Review Board approved this study. Patients with appendicitis with well-circumscribed abscess were indicated to have nonsurgical treatment followed by IA. Between January 2010 and March 2011, patients with PAA underwent emergency surgery because non-surgical treatment strategies were not applied in our center. After performing the first case of non-surgical treatment followed by IA in 2011, non-surgical treatment strategy was adopted between April 2011 and May 2016 (Fig. 1). We excluded patients diagnosed as malignancy (1), who were pregnant (1), or received other combined operations and already had Crohn's disease. Finally, we selected 171 patients.

The diagnosis of complicated appendicitis with abscess was based on the patient's history, physical examination, and imaging modality (computed tomography (CT) or ultrasonography) findings showing a well circumscribed abscess. In cases of negative CT findings and highly suspicious signs and symptoms, ultrasonography was considered for further evaluation. CT was also considered in cases of negative ultrasonography findings. All ultrasonographic examinations and image readings were performed by radiologists. All operations were performed by four surgeons (two residents and two colorectal surgeons). All of them were qualified, experienced surgeons who performed more than 100 cases of appendicitis including complicated appendicitis. All residents performed surgery under the supervision of a colorectal surgeon.

In non-surgical treatment, patients with PAA were received intravenously with a combination of two antibiotics $\left(3^{\text {rd }}\right.$ generation cephalosporin and metronidazole) until the afebrile, normalization of white blood cell count and $\mathrm{C}$-reactive protein (CRP). Percutaneous drainage (PCD) can be inserted if

2,392 Underwent surgery for acute appendicitis

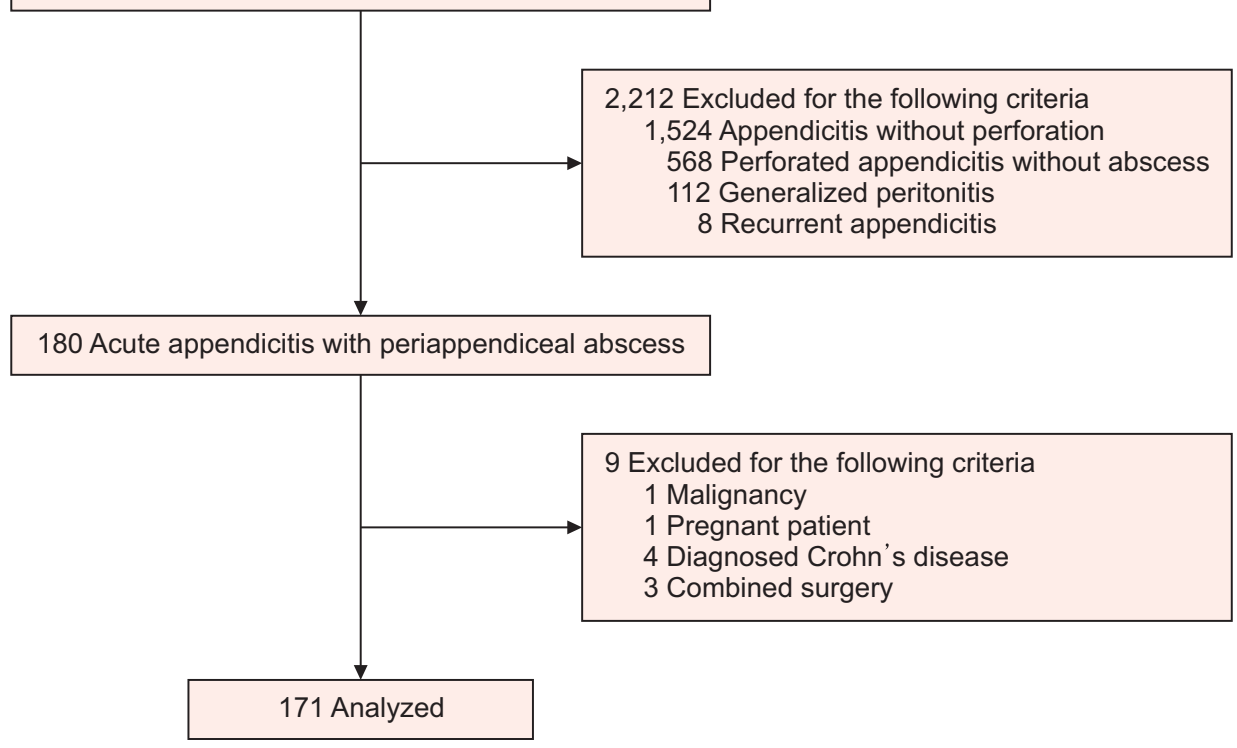

Fig. 1. Patient selection and study flow chart. 
anatomically applicable. Patients were discharged when pain and a soft diet was tolerated. After 4 weeks of discharge, abdominal CT scan was performed to evaluate the improvement of the abscess and to exclude malignancy. Usually, an interval appendectomy was performed after 6 to 8 weeks of discharge.

Patient characteristics that were analyzed were age, gender, presence of significant comorbidities (i.e., diabetes mellitus, hypertension, coronary heart disease, respiratory disease, nephropathy and liver cirrhosis), history of previous abdominal surgery, duration of initial presenting symptoms, and operative time.

Surgical outcomes were determined by postoperative followup. To evaluate the rate of adverse events, we examined the complication rate occurring within 30 days and the readmission rate. Complications were defined as all events necessitating diagnostic or therapeutic measures that increased the length of stay, or required a patient to visit the outpatient clinic on more than two occasions, or led to readmission. Readmission was defined as repeated hospitalization caused by appendectomy-induced complications. Postoperative complications included wound infections, intra-abdominal abscess, intestinal obstructions, paralytic ileus, and cardiovascular problems. Intra-abdominal abscess was confirmed by percutaneous drainage or either CT scan or ultrasonography. Postoperative complications were graded according to the Clavien-Dindo classification.

Postoperative hospital stay was defined as the period of admission for appendectomy.

The main analyses were based on comparisons of patients who experienced postoperative complication with patients who had no complication after surgery, and patients who had different procedures (ES vs. IA). The chi-squared test and $\mathrm{Stu}^{-}$ dent's t-test were used for univariate analyses for categorical and continuous variables. Multivariate logistic regression models were used to identify independent predictors of morbidity following surgery. Factors with a $p$ value $<0.05$ on univariate analysis were incorporated into a multivariate analysis. Statistical significance was defined as a $p<0.05$. Statistical analysis was done using SPSS 21.0 software (SPSS, Chicago, IL, USA).

\section{RESULTS}

The study population included 94 males (54.9\%) and $77 \mathrm{fe}^{-}$ males (45.1\%). The median of 171 patients in the study group was 47 years (range, 5 92 years). The median postoperative hospital stay was 5 days (range, 2 37 days).

At the time of the surgery, 62 (36.2\%) patients had comorbidities including hypertension, diabetes mellitus, chronic renal disease, arrhythmia, and other cardiovascular problems. Hypertension was the most common pre-existing condition (46.0\% of comorbidities), followed by diabetes mellitus, nephropathy and coronary artery disease.

In 171 patients, 28 (16.4\%) developed postoperative complications. Incidence and type of postoperative complications in patients with PAA are shown in Fig. 2. There was no mortality. In the Clavien-Dindo classification, grade II complications (7.0\%) were the most common, followed by grade III (5.3\%) and grade I (4.1\%). There were no grade IV or V complications. Of the 28 patients who experienced postoperative complications, wound infection (7.6\%) had the highest incidence in

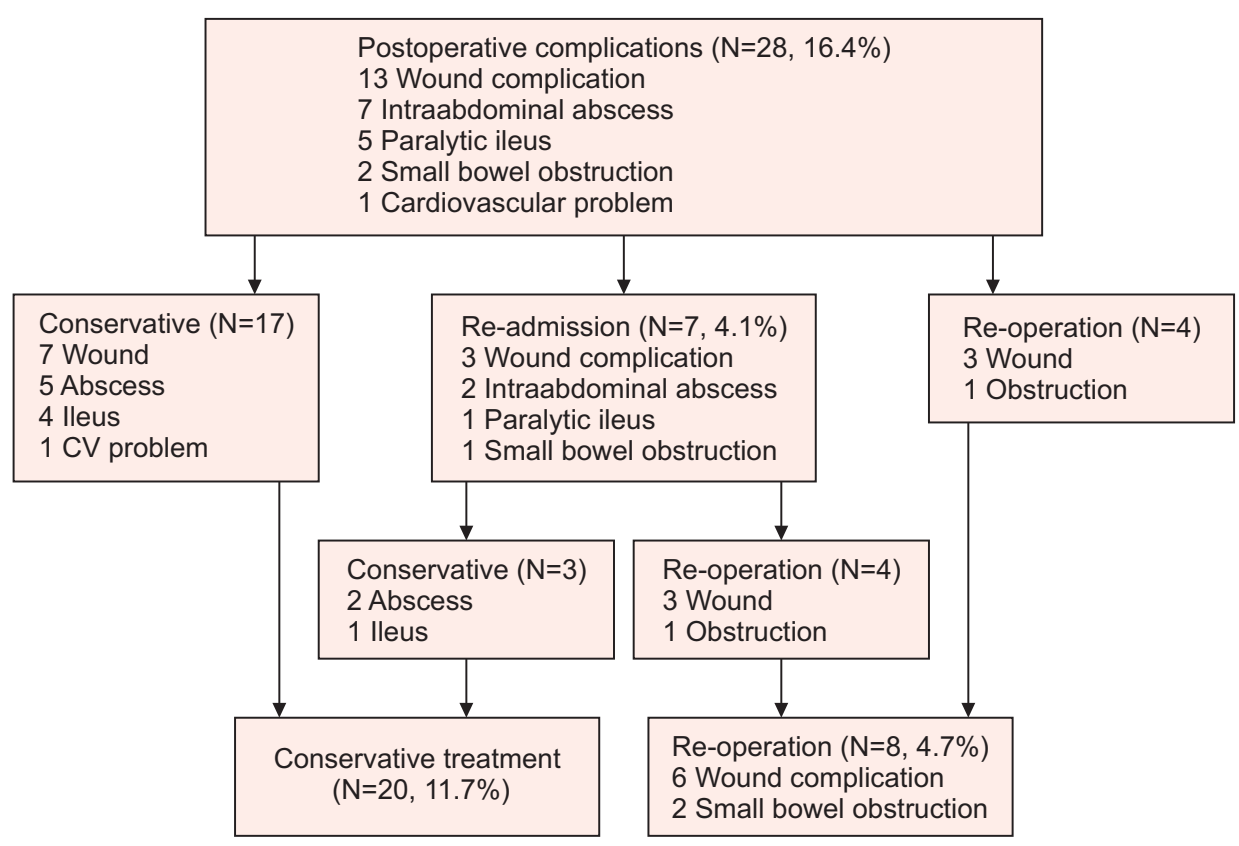

Fig. 2. Incidence and type of postoperative complications in patients with periappendiceal abscess (flow chart). 
the total patient, followed by intra-abdominal abscess (4.1\%), paralytic ileus (2.9\%), and small bowel obstruction (1.2\%). More rarely, small bowel obstruction caused by postoperative adhesion and cardiovascular problem (atrial fibrillation) occurred. Seven patients (4.1\%) were re-admitted with wound complications (1.8\%), intra-abdominal abscess (1.2\%), paralytic ileus $(0.6 \%)$, and small bowel obstruction ( $0.6 \%)$, respectively. There were 8 patients $(4.7 \%)$ who underwent reoperation and, four of them received reoperation during hospitalization before discharge. Six (3.5\%) patients developed complications that required reoperations due to wound complication (wound infection and dehiscence). And two (1.2\%) patients required reoperation due to small bowel obstruction.

Comparisons of demographics and preoperative charac-

Table 1. Demographic and clinical characteristics

\begin{tabular}{lccc}
\hline \multicolumn{1}{c}{ Characteristics } & \multicolumn{3}{c}{ No. of patients (\%) } \\
\cline { 2 - 4 } & $\begin{array}{c}\text { No complication } \\
(\mathbf{n}=143)\end{array}$ & $\begin{array}{c}\text { Complication } \\
(\mathbf{n}=28)\end{array}$ & $p$ value ${ }^{\text {a }}$ \\
\hline $\begin{array}{c}\text { Age (mean } \pm \text { standard } \\
\text { deviation) }\end{array}$ & $46.5 \pm 21.1$ & $44.7 \pm 21.6$ & 0.681 \\
Age $\geq 60$ years) & $39(27.3 \%)$ & $5(17.9 \%)$ & 0.353 \\
Gender (male) & $80(55.9 \%)$ & $14(50.0 \%)$ & 0.679 \\
Comorbidity & $55(38.5 \%)$ & $7(25.0 \%)$ & 0.203 \\
Cardiovascular disease & $41(28.7 \%)$ & $6(21.4 \%)$ & 0.496 \\
Previous operative history & $22(15.4 \%)$ & $2(7.1 \%)$ & 0.375 \\
PCD insertion & $13(9.1 \%)$ & $1(3.6 \%)$ & 0.471 \\
Symptom & & & \\
$\geq 3$ days & $115(80.4 \%)$ & $21(75.0 \%)$ & 0.608 \\
$\geq 5$ days & $78(54.5 \%)$ & $17(60.7 \%)$ & 0.678 \\
$\geq 7$ days & $61(42.7 \%)$ & $12(42.9 \%)$ & 1.000 \\
Abscess & & & \\
$\geq 3$ cm & $92(64.3 \%)$ & $18(64.3 \%)$ & 1.000 \\
$\geq 5$ cm & $42(29.4 \%)$ & $7(25.0 \%)$ & 0.820 \\
$\geq 7$ cm & $17(11.9 \%)$ & $5(17.9 \%)$ & 0.366 \\
\hline Surgical approach & & & \\
Open surgery & $57(72.2 \%)$ & $6(6.5 \%)$ & $<0.001$ \\
Laparoscopic surgery & $86(93.5 \%)$ & $22(27.8 \%)$ & \\
Treatment method & & & \\
Emergency surgery & $62(43.4 \%)$ & $25(89.3 \%)$ & $<0.001$ \\
Interval appendectomy & $81(56.6 \%)$ & $3(10.7 \%)$ & \\
\hline & & & \\
\hline
\end{tabular}

${ }^{a}$ Cross-table analysis using Fisher's exact test; $\mathrm{PCD}=$ percutaneous drainage. Bold print, $p<0.05$. teristics between patients with and without complications are shown in Table 1. Univariate analysis revealed that preoperative factors significantly associated with postoperative complication were open surgery and ES ( $p<0.001$, both). No other preoperative factor was associated with an increased risk of postoperative complication, including patient age, sex, comorbidities, past operative history, and use of percutaneous drainage. There were no statistical differences in duration between the first presentation of symptom and operation (three or more days; five or more days; seven or more days). There was also no difference in the diameter of intra-abdominal abscess in CT $(\geq 3 \mathrm{~cm} ; \geq 5 \mathrm{~cm} ; \geq 7 \mathrm{~cm})$. In multivariate analysis, ES was identified as the only independent factor of postoperative complication (Relative risk, 15.0; 95\% Confidence interval, 2.4 92.5) (Table 2).

Operative characteristics and postoperative outcomes according to the treatment strategies (IA and ES), that display significant differences included laparoscopic approach, postoperative hospital stay, total antibiotics use, and postoperative use of antibiotics (Table 3). The postoperative complication rate of patients with PAA in ES group was 28.7\%, which was statistically higher than that of IA group (3.6\%). Especially, laparoscopic surgery was performed more frequently in the IA group. There was only one case (1.2\%) of open conversion due to severe adhesion in the IA group, which was the only open case in the IA group. Of the 17 cases that were initiated with laparoscopic surgery in the ES group, 8 cases (47.1\%) were converted to open surgery. Among these parameters, except for total antibiotics use, negative outcomes were evident in the ES group. Although the number of days of antibiotics use after surgery was statistically significantly shorter in the IA group $(p<0.001)$, the total number of days of antibiotics use including the preoperative admission period was longer in the IA group $(p<0.001)$. There was no statistically significant difference between the two groups during the entire hospitalization period, although there was additional preoperative admission period for antibiotic treatment in the IA group $(9.7 \pm 4.2$ vs. $9.7 \pm 5.6$, $p=0.949)$ (Table 3).

Table 2. Multivariate analysis of risk factors

\begin{tabular}{lccc}
\multicolumn{1}{c}{ Parameters } & Relative risk & $\begin{array}{c}95 \% \text { confidential } \\
\text { interval }\end{array}$ & p value $^{\mathrm{a}}$ \\
\hline Age $(\geq 60$ years $)$ & 2.992 & $0.928 \sim 9.642$ & 0.066 \\
Gender (male) & 0.667 & $0.275 \sim 1.616$ & 0.370 \\
Open surgery & 1.306 & $0.293 \sim 5.819$ & 0.726 \\
Emergency surgery & 15.074 & $2.456 \sim 92.505$ & 0.003 \\
\hline
\end{tabular}

${ }^{a}$ Multivariate analysis using the binary logistic regression analysis. Bold print, $p<0.05$. 
Table 3. Operative characteristics and postoperative outcomes according to the treatment strategies

\begin{tabular}{|c|c|c|c|}
\hline \multirow{2}{*}{ Parameters } & \multicolumn{3}{|c|}{ No. of patients $(\%)$} \\
\hline & $I A^{\mathrm{a}}(\mathrm{n}=84)$ & $E S^{b}(n=87)$ & $p$ value $^{c}$ \\
\hline Operative time (min) & $64.1 \pm 30.5$ & $106.6 \pm 41.3$ & $<0.001$ \\
\hline Laparoscopy (number) & $83(98.8)$ & $9(10.3)$ & $<0.001$ \\
\hline Hospital stay for non-surgical treatment & $6.9 \pm 3.5$ & 0 & - \\
\hline Postoperative stay (day) & $2.7 \pm 1.6$ & $9.7 \pm 5.6$ & $<0.001$ \\
\hline Total stay (day) & $9.7 \pm 4.2$ & $9.7 \pm 5.6$ & 0.949 \\
\hline Postoperative antibiotics use (day) & $7.8 \pm 2.8$ & $12.6 \pm 8.8$ & $<0.001$ \\
\hline Total antibiotics use (day) & $26.3 \pm 10.3$ & $12.6 \pm 8.8$ & $<0.001$ \\
\hline Re-admission & $0(0.0)$ & $7(8.0)$ & 0.008 \\
\hline Re-operation & $0(0.0)$ & $8(9.2)$ & 0.004 \\
\hline Bowel resection & $1(1.2)$ & $4(4.6)$ & 0.186 \\
\hline Extended incision & $0(0.0)$ & $5(5.7)$ & 0.026 \\
\hline Postoperative complication & $3(3.6)$ & $25(28.7)$ & $<0.001$ \\
\hline Wound complication & $3(3.6)$ & $10(11.5)$ & 0.046 \\
\hline Intra-abdominal Abscess & 0 & $7(8.0)$ & 0.008 \\
\hline Paralytic ileus & 0 & $5(5.7)$ & 0.026 \\
\hline Mechanical obstruction & 0 & $2(2.3)$ & 0.162 \\
\hline Cardiovascular problem & 0 & $1(1.1)$ & 0.324 \\
\hline Clavien-Dindo Classification ( $\geq 3$ ) & 0 & $9(10.5)$ & 0.002 \\
\hline Postop hospital visit (time) & $1.1 \pm 0.7$ & $1.6 \pm 1.4$ & 0.008 \\
\hline
\end{tabular}

${ }^{\mathrm{a}} \mathrm{A}=$ Interval appendectomy; ${ }^{\mathrm{b}} \mathrm{ES}=$ Emergency surgery; ${ }^{\mathrm{C} C}$ Cross-table analysis using Fisher's exact test. Bold print, $p<0.05$.

A

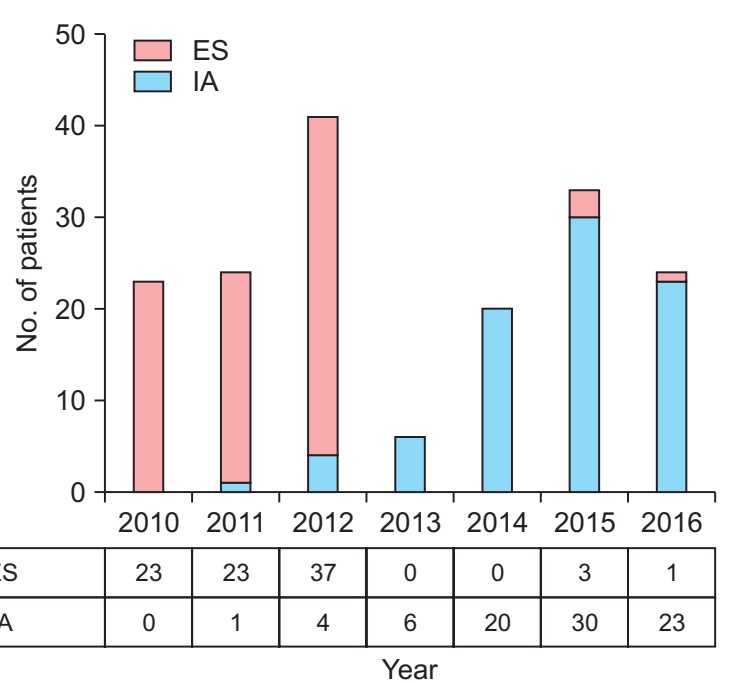

B

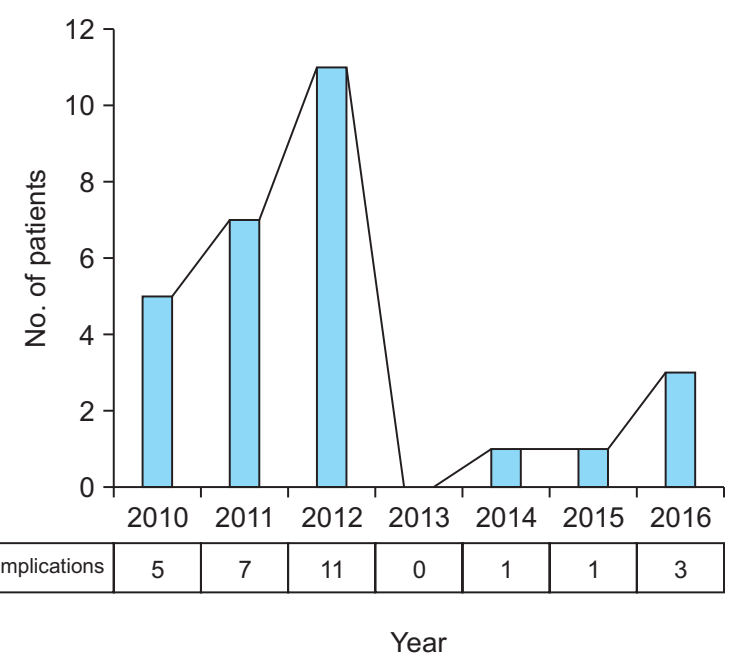

Fig. 3. Annual variation in the number of postoperative complications according to treatment strategy. (A) Number of annual surgeries for patient with periappendiceal abscess. (B) Number of annual postoperative complications in patients with periappendiceal abscess. ES = emergency surgery; $I A=$ interval appendectomy. 
The annual variation in the number of postoperative complications according to treatment strategy is shown in Fig. 3. The annual number of ES and IA are shown in Fig. 3A. Beginning with the first case in 2011, we performed IA for all patients who had perforated appendicitis with PAA in 2013 and 2014, and also performed IA for the most of patients similarly diagnosed disease in 2015 and 2016. The number of postoperative complications was higher in 2011 and 2012, with no complications experienced in 2013 (Fig. 3B). The number of postoperative complications was dramatically decreased after the beginning in earnest of IA for the patients who had perforated appendicitis with PAA in 2013.

\section{DISCUSSION}

In our study of 171 consecutive patients who underwent surgery for PAA, 28 (16.4\%) experienced complications within 30 days of surgery. Wound infection and intra-abdominal abscess were the most common postoperative complications. Only ES was associated with postoperative complications in univariate and multivariate analysis.

Emergency surgery may be technically demanding in patients with PAA or phlegmon formed after acute perforated appendicitis, because of the adhesive loops of the bowel, distorted anatomy, and the difficulties in closing the appendiceal stump due to the inflammation within a wide area of the abdominal cavity. Under these circumstances, appendectomy often requires extensive dissection and may lead to injury of adjacent structures. Complications such as postoperative abscess or enterocutaneous fistula may ensue, necessitating an ileostomy or cecostomy. When an emergency surgery is performed in such patients, the incidence of complications is reported to be up to $36 \% .^{1,11-14}$ In our study, the postoperative complication rate was $28.7 \%$ and reoperation rate was $9.2 \%$ in the IS group which is in line with the published data.

Complicated appendicitis has long been considered a significant factor in longer hospitalization and requirement for extended antibiotic coverage due to its close relationship with higher incidence of postoperative complications. ${ }^{15,16}$ Our study also showed that the length of hospital days and the duration of antibiotics use after IS were protracted (9.7 days and 12.6 days, respectively).

Non-surgical treatment followed by IA has been associated with significantly fewer perioperative complications, such as intraoperative visceral damage, postoperative abdominal and pelvic abscesses, and wound infections, which sometimes require re-intervention. ${ }^{17}$

Non-surgical treatment also allowed a significantly shorter operative time in removing of a diminished inflammatory or normal appendix without adherence than in IA, reducing overall hospitalization time. ${ }^{18}$

In line with the literature, presently there were significantly fewer complications and shorter operative time in nonsurgical treatment followed IA compared with in IS. As shown in Fig. 1 and Fig. 2, the rate of postoperative complications of PAA has decreased dramatically since IA was introduced in our center. In addition, although an additional hospitalization (non-surgical treatment+IA) was needed to treat PAA in non-surgical treatment followed by IA, the overall hospitalization time was not statistically different between the IA and ES groups. Previous studies reported a high success rate of $76 \sim 97 \%$ for non-surgical treatment. ${ }^{2-8}$ In the present study, there were 3 cases $(3.6 \%)$ of treatment failure in non-surgical management. Two of them underwent emergent surgery under septic condition due to deterioration of inflammation despite initial antibiotic treatment. The remaining one was improved after initial antibiotic treatment and discharged, but underwent an operation because the inflammation re-exacerbated while waiting for interval appendectomy.

In particular, the rate of open surgery was much higher in patients undergoing ES with PAA, whereas laparoscopic surgery was performed more frequently in the IA group. In addition, the conversion rate from laparoscopic to open surgery was much higher in the ES group (1.2\% vs. $47.1 \%, p<0.001$ ). With the expectation that the patient will be in danger due to serious postoperative complications, surgeons may have decided to perform open surgery rather than laparoscopic surgery in ES of PAA. In actual practice, not infrequently, even early laparoscopic surgery will be converted to a more extensive open surgical procedure, such as an ileocecal resection or a right hemicolectomy, due to technical problems or distorted anatomy. ${ }^{1,10}$

The development of laparoscopic techniques offers new modalities in the treatment of complicated appendicitis and PAA. However, whether laparoscopic appendectomy should be viewed as the optimal management modality of patients with complicated appendicitis is still a contentious issue. ${ }^{19}$ Several authors have reported significant postoperative complications following laparoscopic appendectomy in patients with PAA, and suggested caution in the use of laparoscopy for complicated appendicitis. ${ }^{20-22}$ The possible reasons for the increased incidence of postoperative intra-abdominal complications might be the spread of localized infection throughout the abdominal cavity during pneumoperitoneum or separation of the appendix within the peritoneal cavity. ${ }^{23}$

However, since PAA are resolved after non-surgical treatment, laparoscopic surgery can be easily applied, and the rate of laparoscopic surgery may increase in IA. The success of laparoscopic IA has been reported without perioperative morbidity and the percentage of IA procedures that included 
laparoscopically has markedly increased in recent years from $30 \%$ to $85 \%{ }^{3,24-26}$ This trend will certainly make it possible to maximize the advantages of laparoscopic surgery for PAA. The advantages include fewer wound infections, less postoperative pain, shorter hospital stay, quicker recovery, and quicker return to normal activities. ${ }^{27-29}$

After successful conservative or/and image-guided drainage management, IA is often not necessary and can safely be omitted, except in patients with recurrent symptoms. ${ }^{1,2,10,30}$ However, proponents of IA point to advantages of initial conservative treatment followed by IA; the approach avoids the recurrence of symptoms and the misdiagnosis of alternative pathologies, such as malignancy, tuberculosis, diverticulosis, and Crohn's disease. ${ }^{9,10}$

In our study period, after non- surgical treatment, one patient had appendiceal cancer, and four had Crohn's disease. Although these patients were excluded from the analyses, we believe that IA after non-surgical treatment should be considered to exclude other pathologic causes of periappendiceal mass, especially in patients older than 40 years old age. In terms of patient safety and stress, we believe that even a slightest risk of recurrent appendicitis with abscess is still a risk for the patients. Alerting the patient to the potential risk of another acute appendicitis episode may trigger fear and anxiety, and excessive surveillance. . $^{31,32}$

This study has several limitations. Owing to its retrospective design and based on a small number of patients in a single institute, we could not be sure whether other factors, such as surgeon's experience and laparoscopic preference, affected the rate of postoperative complications and the rate of laparoscopic surgery. Moreover, the surgeon's assessment of the state of complicated appendicitis at the time of diagnosis is important when deciding whether or not to undergo surgery. This assessment is subjective and difficult to quantify. However, all patients in this study were followed-up in the same unit by the same group of physicians, who used similar guidelines and made decisions collectively. During our study, the Korean Diagnosis-Related Groups (DRG) system was introduced. This system may have affected the number of days after surgery and the median rate of complication. However, when divided into two periods based on the introduction of the DRG system, we found the postoperative complication was still significantly reduced in the interval appendectomy group. So we are confident that the results will not be changed. Cost-effectiveness was not evaluated. Nevertheless, the high clinical effectiveness of IA indicates that it can reduce postoperative complications and the length of hospitalization, and can increase the rate of laparoscopic surgery. In our study, the total antibiotics use were longer in the IA group than in the ES group. The concept of treatment for the number of days of antibiotics use has not yet been established. Further research should be undertaken to determine best practice in the conservative antibiotics management for PAA.

\section{CONCLUSIONS}

Our study shows that patients at the greatest risk of postoperative complication are those who have an emergency surgery for PAA, and the postoperative complication rate is high. Wound infection and intra-abdominal abscess were the most common postoperative complications. Although non-surgical treatment followed by IA has a disadvantage of a longer total antibiotics use, it can reduce the postoperative complication rate and total hospitalization days, and increase the rate of laparoscopic surgery. We recommend that IA should be considered first treatment choice for acute appendicitis accompanied by PAA. Moreover, this result suggests that further prospective randomized controlled multi-institutional trial is needed to decrease the total antibiotics use and evaluate the cost-effectiveness of non-surgical treatment followed by IA in patients with PAA.

\section{REFERENCES}

1) Andersson RE, Petzold MG. Nonsurgical treatment of appendiceal abscess or phlegmon: a systematic review and meta-analysis. Ann Surg 2007;246:741-748.

2) Zerem E, Salkic N, Imamovic G, Terzic I. Comparison of therapeutic effectiveness of percutaneous drainage with antibiotics versus antibiotics alone in the treatment of periappendiceal abscess: is appendectomy always necessary after perforation of appendix? Surg Endosc 2007;21:461-466.

3) Meshikhes AW. Appendiceal mass: is interval appendicectomy "something of the past"? World J Gastroenterol 2011;17:29772980.

4) Zerem E, Imamovic G, Ljuca F, Alidzanovic J. What is the optimal treatment for appendiceal mass formed after acute perforated appendicitis. World J Gastroenterol 2012;18:1849-1850.

5) Meshikhes AW. Management of appendiceal mass: controversial issues revisited. J Gastrointest Surg 2008;12:767-775.

6) Tannoury J, Abboud B. Treatment options of inflammatory appendiceal masses in adults. World J Gastroenterol 2013;19:39423950.

7) Hansson J, Korner U, Khorram-Manesh A, Solberg A, Lundholm K. Randomized clinical trial of antibiotic therapy versus appendicectomy as primary treatment of acute appendicitis in unselected patients. Br J Surg 2009;96:473-481.

8) Hansson J, Korner U, Ludwigs K, Johnsson E, Jonsson C, Lundholm K. Antibiotics as first-line therapy for acute appendicitis: evidence for a change in clinical practice. World J Surg 
2012;36:2028-2036.

9) You KS, Kim DH, Yun HY, et al. The value of a laparoscopic interval appendectomy for treatment of a periappendiceal abscess: experience of a single medical center. Surg Laparosc Endosc Percutan Tech 2012;22:127-130.

10) Brown CV, Abrishami M, Muller M, Velmahos GC. Appendiceal abscess: immediate operation or percutaneous drainage? Am Surg 2003;69:829-832.

11) Ahmed I, Deakin D, Parsons SL. Appendix mass: do we know how to treat it? Ann R Coll Surg Engl 2005;87:191-195.

12) Sadot E, Wasserberg N, Shapiro R, Keidar A, Oberman B, Sadetzki S. Acute appendicitis in the twenty-first century: should we modify the management protocol? J Gastrointest Surg 2013;17:1462-1470.

13) Erdogan D, Karaman I, Narci A, et al. Comparison of two methods for the management of appendicular mass in children. Pediatr Surg Int 2005;21:81-83.

14) Kim JK, Ryoo S, Oh HK, et al. Management of appendicitis presenting with abscess or mass. J Korean Soc Coloproctol 2010; 26:413-419.

15) Fukami Y, Hasegawa H, Sakamoto E, Komatsu S, Hiromatsu T. Value of laparoscopic appendectomy in perforated appendicitis. World J Surg 2007;31:93-97.

16) Sleem R, Fisher S, Gestring M, et al. Perforated appendicitis: is early laparoscopic appendectomy appropriate? Surgery 2009;146:731-737; discussion 737-738.

17) Weber TR, Keller MA, Bower RJ, Spinner G, Vierling K. Is delayed operative treatment worth the trouble with perforated appendicitis is children? Am J Surg 2003;186:685-688; discussion 688-689.

18) St Peter SD, Aguayo P, Fraser JD, et al. Initial laparoscopic appendectomy versus initial nonoperative management and interval appendectomy for perforated appendicitis with abscess: a prospective, randomized trial. J Pediatr Surg 2010;45:236-240.

19) Mallick MS, Al-Qahtani A, Al-Bassam A. Laparoscopic appendectomy is a favorable alternative for complicated appendicitis in children. Pediatr Surg Int 2007;23:257-259.

20) Horwitz JR, Custer MD, May BH, Mehall JR, Lally KP. Should laparoscopic appendectomy be avoided for complicated appendicitis in children? J Pediatr Surg 1997;32:1601-1603.
21) Krisher SL, Browne A, Dibbins A, Tkacz N, Curci M. Intraabdominal abscess after laparoscopic appendectomy for perforated appendicitis. Arch Surg 2001;136:438-441.

22) Markar SR, Blackburn S, Cobb R, et al. Laparoscopic versus open appendectomy for complicated and uncomplicated appendicitis in children. J Gastrointest Surg 2012;16:1993-2004.

23) Cariati A, Brignole E, Tonelli E, et al. [Laparoscopic or open appendectomy. Critical review of the literature and personal experience]. G Chir 2001;22:353-357.

24) Lin HF, Lai HS, Lai IR. Laparoscopic treatment of perforated appendicitis. World J Gastroenterol 2014;20:14338-14347.

25) Groves LB, Ladd MR, Gallaher JR, et al. Comparing the cost and outcomes of laparoscopic versus open appendectomy for perforated appendicitis in children. Am Surg 2013;79:861-864.

26) Senapathi PS, Bhattacharya D, Ammori BJ. Early laparoscopic appendectomy for appendicular mass. Surg Endosc 2002;16:17831785.

27) Esposito C, Borzi P, Valla JS, et al. Laparoscopic versus open appendectomy in children: a retrospective comparative study of 2,332 cases. World J Surg 2007;31:750-755.

28) Yagmurlu A, Vernon A, Barnhart DC, Georgeson KE, Harmon CM. Laparoscopic appendectomy for perforated appendicitis: a comparison with open appendectomy. Surg Endosc 2006;20:10511054.

29) Partrick DA. Prospective evaluation of a primary laparoscopic approach for children presenting with simple or complicated appendicitis. Am J Surg 2006;192:750-755.

30) Wullstein C, Barkhausen S, Gross E. Results of laparoscopic vs. conventional appendectomy in complicated appendicitis. Dis $\mathrm{Co}^{-}$ lon Rectum 2001;44:1700-1705.

31) Iqbal CW, Knott EM, Mortellaro VE, Fitzgerald KM, Sharp SW, St Peter SD. Interval appendectomy after perforated appendicitis: what are the operative risks and luminal patency rates? J Surg Res 2012;177:127-130.

32) Schurman JV, Cushing CC, Garey CL, Laituri CA, St Peter SD. Quality of life assessment between laparoscopic appendectomy at presentation and interval appendectomy for perforated appendicitis with abscess: analysis of a prospective randomized trial. J Pediatr Surg 2011;46:1121-1125. 